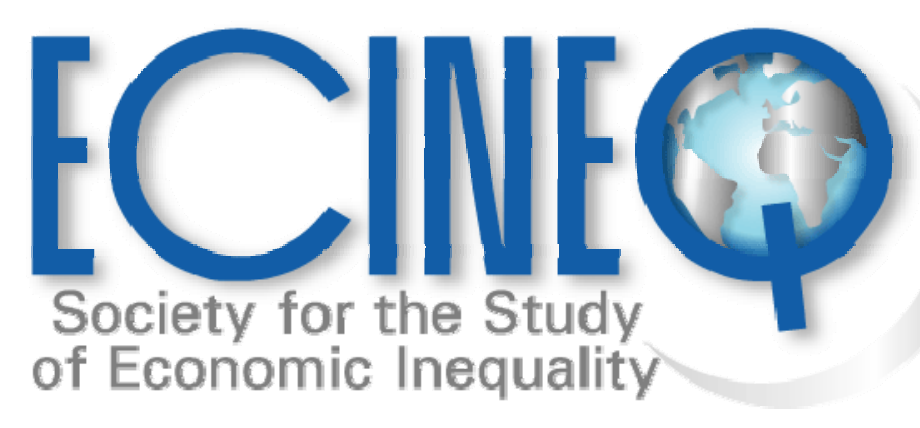

Working Paper Series

A model for microeconomic and macroeconomic development

Ivan O. Kitov 


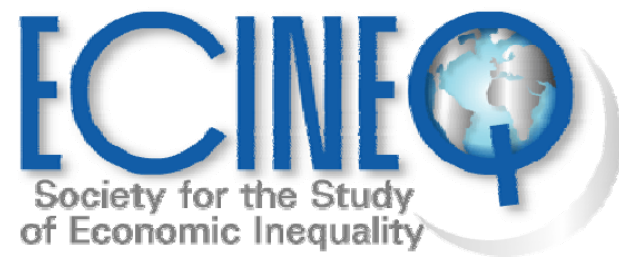

ECINEQ 2005-05

November 2005

www.ecineq.org

\title{
A model for microeconomic and macroeconomic development
}

\author{
Ivan O. Kitov ${ }^{*}$ \\ Russian Academy of Sciences
}

\begin{abstract}
A comprehensive study of the personal income distribution (PID) in the USA is carried out. Principal characteristics of the PID in USA are established. A microeconomic model of the personal income distribution and evolution with time is developed. The model balances two processes individual income earning and dissipation of the income. The model accurately predicts the overall PID and its evolution and fine features of the PID in various age and income groups. The results obtained prove that the observed economic growth is a predetermined process governed only by a country specific single year of age population.
\end{abstract}

KEYWORDS: personal income distribution, microeconomic modeling, real GDP, macroeconomics

JEL Classification: D01, D31, E17, J1, O12

* Contact details: VIC, P.O. Box 1250, Vienna 1400, Austria; email - ikitov@mail.ru 


\section{Introduction}

An interdisciplinary approach has shown its fruitfulness in many scientific and technological areas. This success is not only a result of coincidence of formal description of various physical, chemical, biological, and sociological processes, but also expresses an existence of very deep common roots. For example, a power law size distribution is observed in economics (Pareto distribution), frequencies of words in longer texts, seismology (Guttenberg-Richter recurrence curve), geomechanics (fractured particle sizes), the standardized and many other areas. Recent study conducted by Lise and Paczuski (2002) refers the power law distribution to a realization of some stochastic processes known as a self-organized criticality (SOC).

Economics as a science and its numerous applications in real life demands a huge amount of numerical data in order to estimate the current state and probability of future development. The data have been continuously gathered from the very beginning of capitalism as a system, but the $20^{\text {th }}$ century and especially its second part is characterized by a burst of economic observations and measurements. The resulting data set became an object of a thorough study not only for professional economists but also for specialists in many other disciplines. There are numerous examples of successful application of mathematical and physical methods from many adjacent disciplines for understanding economic phenomena and processes.

The personal income distribution (PID) represents one of the best sets of numerical data with a history of more than fifty years of continuous measurement with increasing accuracy. Even a simple plotting of the income data reveals some specific features, which are often observed in physics - growth and roll-off is well interpolated by exponential and power functions, i.e. the functions which are solutions of the simplest ordinary differential equation. This makes it very attractive to apply standard methods of analysis and to model income development processes according to 'first principles' adopted in natural sciences.

A model of a solid with inhomogeneous inclusions has been proposed and developed by V.N.Rodionov and co-authors (1982). The model describes behavior of a real solid by separating elastic and inelastic stresses. The latter are concentrated in the inhomogeneous inclusions and play an important role in deformation and fracturing process. The inclusions are distributed in size in such a way that total volume of the inclusions of every size is constant. The growth rate of inelastic stresses concentrated in the inclusions is proportional to deformation rate. The dissipation rate of the inelastic stresses is inversely proportional to the inclusion size. Only one deformation process with a constant rate is considered in the geomechanical model. The deformation is caused by some external forces, which provide a constant energy inflow in the solid. 
This original geomechanical model has been adopted and modified for economic purposes. Formally, the inclusion size is interpreted as a size of some tool or means, which is used to generate income. The means' sizes are evenly distributed over an economy from a nonzero minimum to some finite maximum value. In the economic model, the deformation caused by some external forces is interpreted as a capability of a person to generate income by using some means. The income earned (analog of the inelastic stress concentrated on an inhomogeneous inclusion) is proportional to a product of the means used and the personal capability to earn the income. Unlike the geomechanical model, the capabilities (or deformation rates) are also evenly distributed over the population and the means size increases in time as the personal incomes grow. The resulting economic balance equation includes some features additional to those of the geomechanical model, but has the same functional dependence between the variables and the same formal solution.

The proposed model is a microeconomic model in the sense of addressing evolution of the personal income depending on personal conditions. On the other hand, when integrated over the whole population eligible to receive income, the model provides a macroeconomic consideration. The sum of all the personal incomes (irrelevant to the way they are obtained - employee compensation or personalized profit) is called Gross Domestic Income (GDI) and by definition is equal to GDP. Here we assume that there is no impersonal income because any income, personal or corporate, ultimately has its personal owner who can use the income for consumption, saving or investment. In the following, we do no make any difference between GDP and GDI.

In the model, we trace the continuous evolution of each and every personal income or the personal income trajectory. At any time, the sum of a complete set of personal incomes in a given economy is equal to the current GDP value defined as the total income generated by the production of the goods and services within the economy. Thus, the only source of GDP is the personal incomes, which are continuously changing (growing or decreasing) depending of overall economic conditions. The conditions will be discussed in detail in the following papers, which are currently in preparation. Here we use the real GDP growth rate in the USA (positive or negative) as an external and independent parameter.

It is obvious, that such an approach addressing the personal incomes evolution is completely equivalent to the conventional economic approach, where the total income or GDP is divided into personal incomes and corporate profits. Unfortunately, the current procedure of the personal income measurements artificially excludes the corporate profits (which can be easily personalized) from the published personal income distributions. This is a potential source of distortion for the observed PID, especially in the middle and higher income ranges.

The conventional approach dividing GDI into personal and corporate ones induces some confusion because of a high variance of the total income division between people and companies. It occurred to be very hard to follow these variations and explain them. The personal income distribution, however, evolves in time according to a very simple law revealed below and is completely predictable. Equivalence of the conventional economic approach and that developed below in the sense of addressing the total income leads to a conclusion that the economic growth is predictable and predefined by the current economic state and population age distribution to the degree the personal income distribution is predictable.

This is the first from a series of papers devoted to the microeconomic and macroeconomic modelling of the personal income distribution in the USA. The current paper formally describes the microeconomic model for the personal income evolution. Some specific results obtained from the modelling are presented in the following papers. Those include the modelling of the overall personal income distribution and its evolution in time, 
the modelling of the PID in various age groups and their behaviour in time, and the modelling of the mean personal income dependence on work experience and the evolution of this dependence in time from 1967 to 2003. An additional paper presents some results of the prediction of the value of work experience corresponding to the peak mean income as a function of time. This value is claimed to be a reciprocal value of the observed economic trend in the USA from 1930 to 2003.

\section{Microeconomic model of the personal income distribution and evolution}

The following section is devoted to the development of a microeconomic and macroeconomic model explaining behavior of the personal income distribution in relation to some measured macroeconomic and demographic parameters. A standard procedure is used for the model validation: this involves comparison of the predicted and observed data.

The principal assumption of the model is that every person above fifteen years of age (the official starting age for work) has a capability to work or earn money using some means, which can be a job, bank interest, stocks, etc. An almost complete list of the means is available in the ASEC technical documentation published by the US Census Bureau (1996). The model allows additional redistribution of the income inside households. This effectively includes in consideration all the people above 15 years of age in contrast to the survey procedure, which excludes all those who can not give a positive answer to the questions about earnings included in the survey questionnaire. The number of the people without income is also included in the published income tables and used in the model. The model does not allow anybody to survive without income. So, everybody older than 15 years of age has some positive income.

The total amount of money a person earns is proportional to her/his capability to earn money, s. Here we do not allow any difference between the amount of money earned by the person and the amount of money produced by the person. The amount of money received by the person or the personal income is exactly equal to the amount of money (or total price) the person had produced for the outer world in form of goods and/or services. In this case, the total sum of all the personal incomes in the country is exactly equal to the country GDP. It also means that there is no other source of the personal income except the working age population (all people above 15 years of age).

The person is not isolated from the surrounding world and the work (money) s/he produces dissipates (leaks) in the interaction with the outer world, decreasing the final income. One can consider this dissipation process as related to the effects of the real (physical) world restricting capability to produce and concurrence with the people around who tend to diminish merits or price of the person's product. As in many physical models, the rate of the dissipation is proportional to the attained income level and inversely proportional to the size of the means used to earn the money. One can write a simple balance equation for a person earning money:

$$
d M(t) / d t=s(t)-\alpha M(t) / L(t)
$$

where $M(t)$ is money income, $t$ is the work experience, $s(t)$ is the capability to earn money; and $\alpha$ is the dissipation coefficient. The money income, $M(t)$, is denominated in dollars, the capability $s$ is measured in [\$/year], the earning means size, $L(t)$, has a dimension of [\$], and the dissipation factor, $\alpha$, is measured in [year/\$]. The general solution of the above equation, if $s(t)$ and $L(t)$ are considered to be slowly changing in time external and independent parameters, is as follows (where $s$ and $L$ constants): 


$$
M(t)=(s / \alpha) L(1-\exp (-\alpha t / L))
$$

One can introduce a modified and dimensionless capability to earn money $S(t)=s(t) / \alpha$. The capacity, i.e. the product $S(t) L(t)$, of a "theoretical" person evolves with time as real GDP per capita because the absolute value of the capability and size of the earning means evolves with time as the square root of per capita real GDP.

Relative effective capability to earn money, $S$, and relative size of earning means, $L$, are represented as a sequence of integer numbers evenly distributed over the range from 2 to 30. This range results from a calibration process. The probability for a person to use a means of size $L$ is also a constant over the means i.e. does not depend on capability $S$ and vice versa. Thus, the relative capacity of a person to earn money is distributed as a product of $S$ and $L$, where $S$ and $L$ are independent and take values from 2 to $30: S L=\{2 \times 2,2 \times 3, \ldots$, $2 \times 30,3 \times 2, \ldots, 3 \times 30, \ldots, 30 \times 30\}$.

All the absolute values of $S$ and $L$ evolve with time as square root of the per capita GDP what effectively keeps the relative values constant. Below we implicitly assume all $S$ and $L$ to be taken as absolute values explicitly using in the distribution of their relative values, i.e. $L=30$ means 30 times total increase of the square root of the per capita GDP from some initial time to the given moment.

Figure 1 depicts some examples of the personal income evolution. The curves are normalized to the maximum possible model income $S L=30 \times 30=900$. The dissipation factor is $\alpha=0.07$. The predicted income for a person using a means of size $L=2 / 30$ (normalized to the maximum size of the earning means) and having a capability to earn money $S=2 / 30$ (normalized to the maximum capacity to earn money) approaches the maximum level of $4 / 30^{2}$ (relative to the overall maximum possible of $30^{2} / 30^{2}=1$ ) several years after work starts. During the rest of her/his life, the person has the same relative personal income, and the absolute value of the income increases proportionally to the per capita real GDP growth.

In the case where $S=15 / 30$ and $L=15 / 30$, a longer time is necessary for a person to approach the maximum potential income equal to $15^{2} / 30^{2}$. This person reaches $95 \%$ of the potential income in 10 to 15 years from the start of work. Then almost no change of relative income is observed as in the case of the lower income person. The absolute income, however, increases with time as the real GDP per capita.

It is interesting to compare two cases with the same potential maximum level of income but different $L$ values. These cases are $S=2 / 30, L=30 / 30$ and $S=30 / 30, L=2 / 30$. The corresponding curves in Figure 1 reach the same level in 40 years and approximately 3 years, respectively. Hence, the earning means' size plays a key role in the evolution of the PID. This parameter defines the change of dissipation rate in equation (2), because $\alpha$ is a constant. The effective time constant in (2) is $L / \alpha$. The larger the effective time constant is the longer time is needed to reach the same relative level of income. So an $L$ increase leads to a slower relative income growth.

One can not determine the numerical value of the dissipation factor by independent measurements. Thus, a standard calibration procedure is applied. The absolute value of $L$ is defined as equal to $30 / 30=1.0$ at the start point of the studied time period. Then $\alpha$ is varied to match the predicted and observed personal income distributions (PIDS), and the best fit value of $\alpha$ is used for further predictions. Figure 2 presents some examples of the income evolution for various effective values of $\alpha$ ranging from 0.09 to 0.04 . This range is approximately the same as obtained in the modelling the PID for the time period from 1960 to 2002. The absolute value of $L$ changes during this period from 1.0 to 1.49 according to the per capita real GDP total growth by a factor of 2.22 for the period. One can see that more 
and more time is necessary for a person with $S=L=30 / 30$ to reach the lower limit of the income range where incomes are distributed according to the Pareto law (called the Pareto threshold) as shown in Figure 3. The curve is obtained from (2) with the assumption that $M(t)=0.43$. The equation is converted into the form $T_{c}=\ln (1-0.43) L / \alpha$, where $T_{c}$ is the time period of the income growth from zero to the Pareto threshold. Thus, $T_{C}$ (for $S=L=30 / 30$ ) is proportional to the square root of the per capita real GDP growth.

The Pareto distribution threshold normalized to the maximum possible model income of 900 is 0.43 . When a person reaches this limit, s/he potentially can reach any income with a probability described by the power law distribution. This approach is borrowed from the modern natural sciences studying self organized criticality (SOC) which "lead to scale free, e.g. power law, distribution of sizes and is normally related to some long range spatial and temporal correlation within the system. Typical natural realizations of this phenomena include, among others, earthquakes, forest fires, and biological evolution" (see Lise and Paczuski (2002)). The value of 0.43 is also obtained by a calibration matching the observed number of people governed by Pareto distribution and the corresponding predicted number. Only people with high $S$ and $L$ can eventually reach the threshold as shown in Figure 4.

If the money earning capacity, $S L$, drops to zero at some critical time in a personal history, $T_{c r}$, the solution is

$$
M(t)=M\left(T_{c r}\right) \exp \left(-\alpha\left(t-T_{c r}\right) / L\right)=S L\left(1-\exp \left(-\alpha T_{c r} / L\right)\right) \exp \left(-\alpha\left(t-T_{c r}\right) / L\right)
$$

The first term is equal to the income value attained by a person at time $T_{c r}$, and the second term presents the exponential decay of the income above $T_{c r}$. Thus, the observed exponential roll-off of the mean income beyond $T_{c r}$ corresponds to zero work in the model. People do not exercise any effort to produce income starting from some predefined point in time, $T_{c r}$, and enjoy exponential decay of their income. Figure 5 illustrates schematically solutions (2) and (3). A normalized mean income distribution in the USA for 2002 is interpolated by two functions: $\left(1-\exp \left(-\alpha_{0} t\right)\right)$ in the time interval of work experience from 0 to 39 years, and by $\left(1-\exp \left(-\alpha_{0} T_{c r}\right)\right) \exp \left(-\alpha_{1} t\right)$ above this critical work experience. Averaging of the theoretical incomes is conducted in 10 year time intervals to match the observations. The dissipation factor $\alpha_{1}$ is $0.7 \alpha_{0}$ in order to fit the observations. This fact indicates that the process of exponential decay is potentially characterized by a different dissipation coefficient. The interpolation does not present a correct model since it does not distinguish between people with different incomes and does not give the driving force of the income evolution. This is an example of the approximation often used instead of "first principle" model. It demonstrates, however, the simplicity of the underlying processes which lead to the observed personal income distribution.

The distribution of the earning means and personal capabilities is constant in the rage from 2 to 30. The constant distribution was selected as the simplest one and because of a formal coincidence with the size distribution in the original geomechanical model. This choice has proved to be valid. The number of people having a given value of the earning capability is equal to $1 / 29$ of the total number. This is valid for the whole population and for every single year of age. The probability for a person to get an earning means of a given size $L$ is also equal to $1 / 29$. The overall income distribution $S L$ in 0.025 wide income intervals (theoretical income range spans from 0 to 1) is presented in Figure 6. There are two important features of the distribution. It has an oscillating character and quasi-exponential decay with income. The former feature is observed in the original income distribution. Figure 6 also presents the PID in the age group from 60 to 64 years for the calendar years of 1994, 1998, and 2001. Numerous combinations of $S$ and $L$ relative size intervals were tried and the best fit combination was selected. The combination is used in the study. Other 
combinations do not provide the same frequency of oscillations and an overall decay fitting the observed one. The original PID for the age group between 60 and 64 years is used in order to avoid time effect. Because the attained level of income depends on time, the younger age groups' PIDs are disturbed at higher incomes where the peak level is not reached. The older groups are also disturbed by the dissipation processes described above. So, the best age group to match the predicted and observed PIDs happens to be the group from 60 to 64 years of age, where the growth has already finished and the decay does not disturb the distribution much.

There is an important feature of the real PID which is not described by the model yet. The real distribution extends from 0 to several hundred million dollars, and theoretical distribution extends only from $\$ 0$ to about $\$ 100,000$, i.e. the income interval used so far to match the observed and predicted distributions. Incomes of approximately 10 per cent of people above 15 years of age in the USA are distributed according to a power law (Pareto law) starting from some threshold (Pareto threshold) income ranging from about $\$ 30,000$ to $\$ 60,000$ for the years from 1994 to 2003 . A theoretical threshold of 0.43 was introduced above in order to match in the model the number of people distributed according to the Pareto law.

One can expect consistency of the real and theoretical distributions below the Pareto threshold. Above the threshold, the theoretical and real distributions diverge. The former drops with an increasing rate to zero at $\$ 100,000$, and the latter decays inversely proportional to the income cubed. Because the real and theoretical income intervals are different, the total amount of money earned by people in the Pareto income zone differs in the real and theoretical cases. This amount is equal to the integral over incomes above the Pareto threshold or the product of a PID and income. Here one can introduce a concept distinguishing below-threshold (sub-critical) and above-threshold (super-critical) behavior of income earners. In the sub-critical zone, an income earned by a person is proportional to her/his efforts or capacity SL. In the super-critical zone, a person can earn any amount of money between the Pareto threshold and the highest possible income. The probability to get a given income drops with income according to the Pareto law.

The total amount of money actually earned in the super-critical zone is of 1.35 times larger than the amount that would be earned if the incomes are presented by the theoretical distribution, in which any income is proportional to the capacity. This factor of 1.35 is very sensitive to the definition of the Pareto threshold. In order to match the theoretical and observed total amount of the money earned in the super-critical zone one has to formally multiply every theoretical person's income in the zone by the factor of 1.35 . This is the last step to equalize theoretical and observed number of people and incomes in both zones: suband super-critical. It seems also reasonable to assume that the observed difference in distributions in the zones is reflected in some basic difference in the capability to earn money. A numerical expression of this difference is the multiplication factor of 1.35.

So, the model is finalized. An individual income grows in time according to relationship (2) until some critical age $T_{c r}$. Above $T_{c r}$, an exponential decrease according to (3) is observed. Whenever an income is above the Pareto threshold it gains $35 \%$ of its theoretical value. All people are divided into equal 29 groups by the capability to earn money. Any new generation has the same structure relative to the capability as the previous generation. All earning means are also divided into 29 equal groups by size. This provides a permanent structure for the potential personal income distribution. Actual PID depends, however, on the single year of age population distribution. The relative value of an individual income is proportional to the capability to earn money and the size of the earning means. The absolute values of the parameters grow proportional to the square root of the per capita GDP growth rate. Critical time $T_{c r}$ also grows proportional to the square root of the 
per capita GDP growth rate. From independent measurement of the population distribution and GDP one can model the evolution of the PID and other income characteristics.

\section{Discussion and conclusions}

The microeconomic model for the evolution of the personal income is based on a 'first principle' approach expressed as a balance equation between money production and money dissipation. The former is the production of goods and services. The latter is the result of a number of factors acting against the individual production effort and reducing the amount of money earned below its potential level. This model is similar to a number of physical models including the model of a solid with inhomogeneous inclusions.

The model presents a functional dependence for the continuous personal income evolution and attributes any economic growth to the personal efforts to earn money. The process of money earning is absolutely equivalent to the process of the goods and services production. The former approach, however, returns us to the roots of the economic activity because the only source of any income or wealth is the personal effort. Due to natural interactions and inevitable ranking in any society the personal incomes are distributed not in a random, but in a predefined way. The actual PIDs demonstrate a simple functional dependence on the real economic growth.

The presented microeconomic model takes the advantage of recent accurate measurements of the personal income distribution in the USA. There was no such data for the period before 1994 and the individual incomes were thought to be unpredictable. This unpredictability is valid for an individual, if to trace his/her income evolution in time. But any individual can only follow one of the predefined trajectories predicted by the model. If somebody suddenly jumps to a new and higher income value some other individual with the income value equal to the new income of the first person has to drop to first person's income value in order to retain the observed fixed PID. This is a conservation law uncovered by the PID observation and described in more detail in the following paper devoted to the overall PID.

The conventional economic approach lacks these natural roots artificially dividing the total personal income into employee compensation and corporate profits with further (artificial) separation into groups attributed to various types of economic activity: consumption, savings, and investment. Relative importance of these artificially separated parts varies with time depending on individual decisions to split a personal income into these three portions. This decision is based, however, only on the individual effort to earn income and can be random in the sense of incomplete information and wrong interpretation. That's why one can not construct a precise model for the economic evolution expressed in terms of these artificial parameters and any economic policy based on a full control of the artificial parameters ultimately fails. There is nothing except the personal income production in any developed economy and the production is predefined. The PID evolution can be exactly predicted to the extent of the accuracy of the population counting and GDP measurements as we demonstrate in the following papers.

\section{Acknowledgments}

The author is grateful to Dr. Wayne Richardson for his constant interest in this study, help and assistance, and fruitful discussions. The manuscript was greatly improved by his critical review.

\section{References}


1. Lise S., and M. Paczuski (2002) "A Nonconservative Earthquake Model of SelfOrganized Criticality on a Random Graph",cond-mat/0204491 v1, 23 Apr 2002, pp. 1-4.

2. Rodionov, V.N., V.M.Tsvetkov, and I.A.Sizov (1982) "Principles of Geomechanics" Nedra, Moscow, p.272. (in Russian)

3. U.S. Census Bureau (1996). “CPS Help-Census/DSD/CPSB” Last modified: May 22, 1996, http://www.bls.census.gov/cps/ads/shistory.htm 
Figures

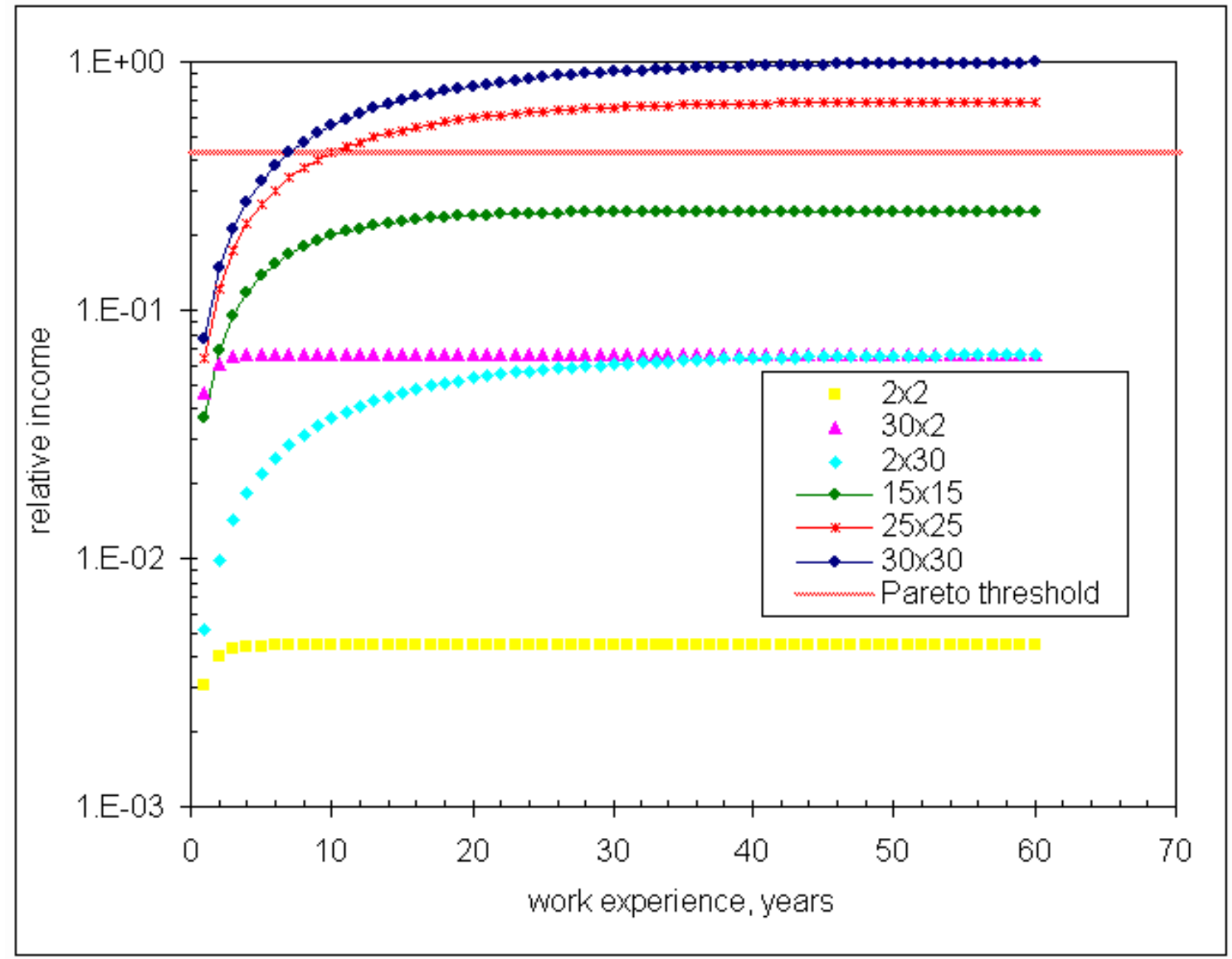

Fig. 1. Evolution of personal income in time for various combinations of earning means size, $\mathrm{L}$, and personal capability to earn money, $\mathrm{S}$. The Pareto distribution threshold is 0.43 . Only people with high $\mathrm{S}$ and $\mathrm{L}$ can eventually reach the threshold. The duration of period needed to reach the maximum income depends on L. Compare cases $30 \times 2$ and $2 \times 30$. Because of a larger $\mathrm{L}$ value of 30 , the first person reaches maximum much faster the second person with the means of size 2 . The dissipation factor $\alpha=0.07$ 


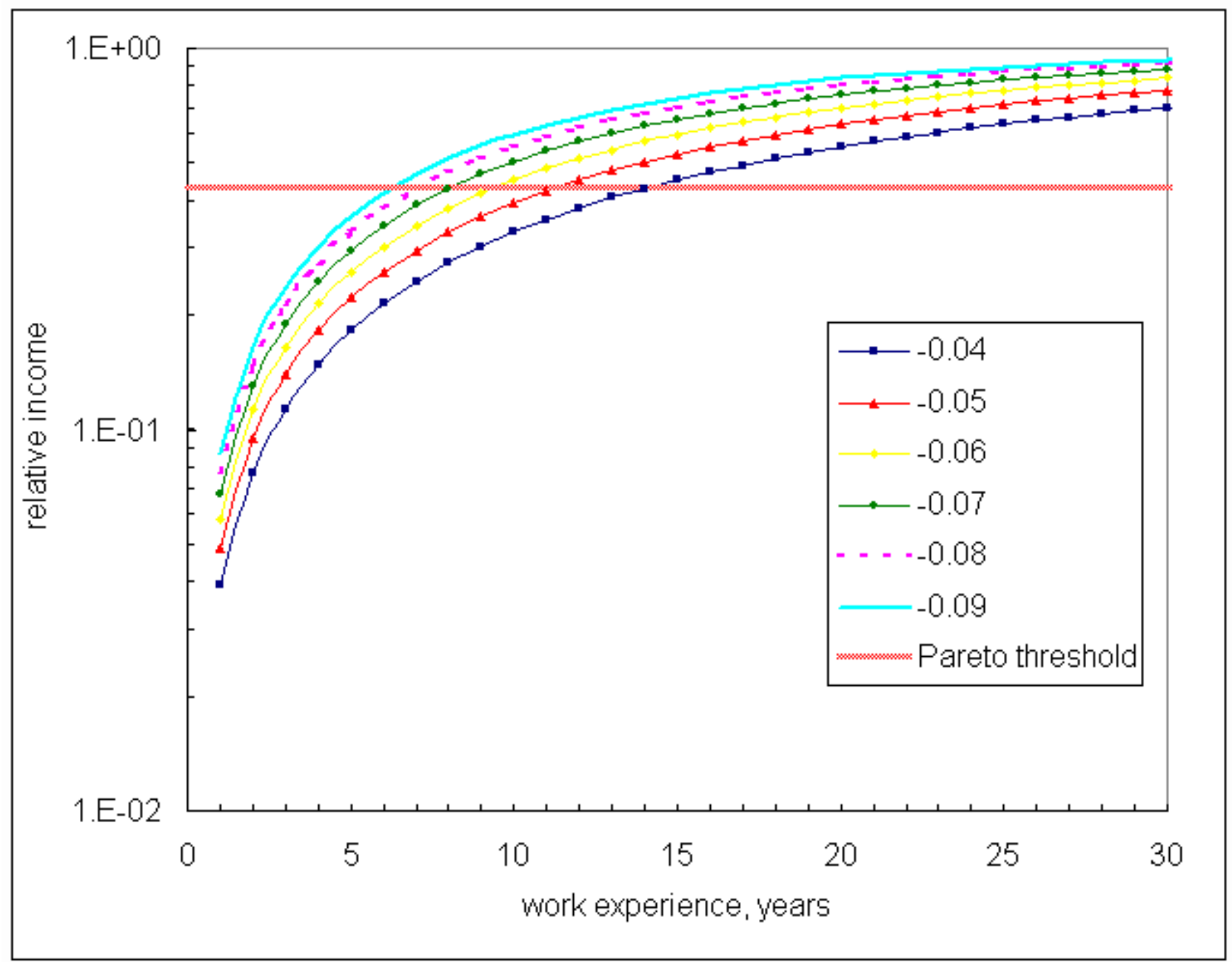

Fig. 2. Evolution of personal income in time for various dissipation factors $\alpha$. The earning means size $L=30$, and personal capability to earn money $S=30$. The Pareto distribution threshold is 0.43 . The duration of period needed to reach maximum income depends on $\alpha$ : the larger $\alpha$ the shorter time is needed to reach Pareto threshold. 


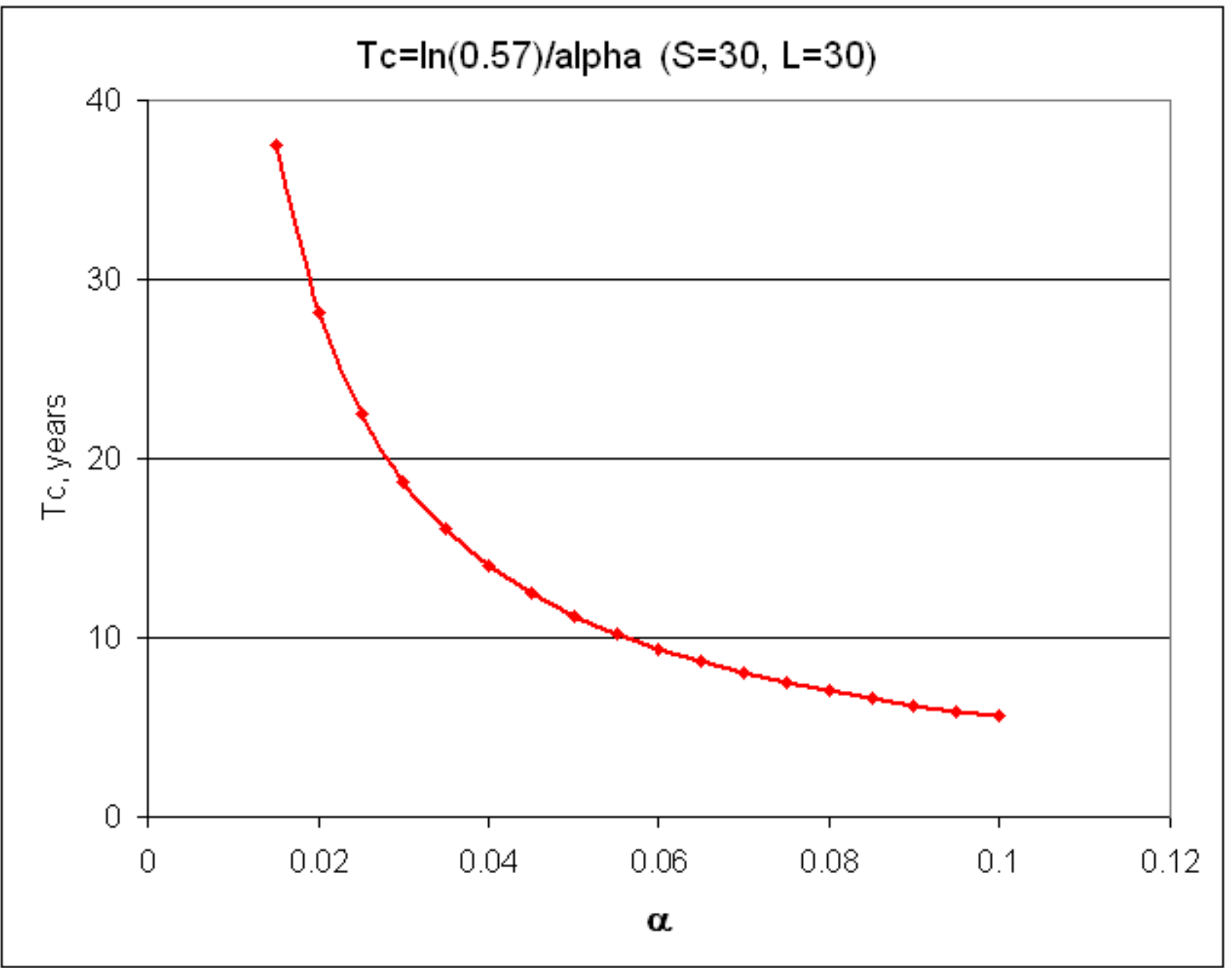

Fig. 3. Time needed to reach Pareto threshold as a function of the dissipation factor $\alpha$. The earning means size $L=30$, and personal capability to earn money $S=30$. The Pareto distribution threshold is 0.43 . 


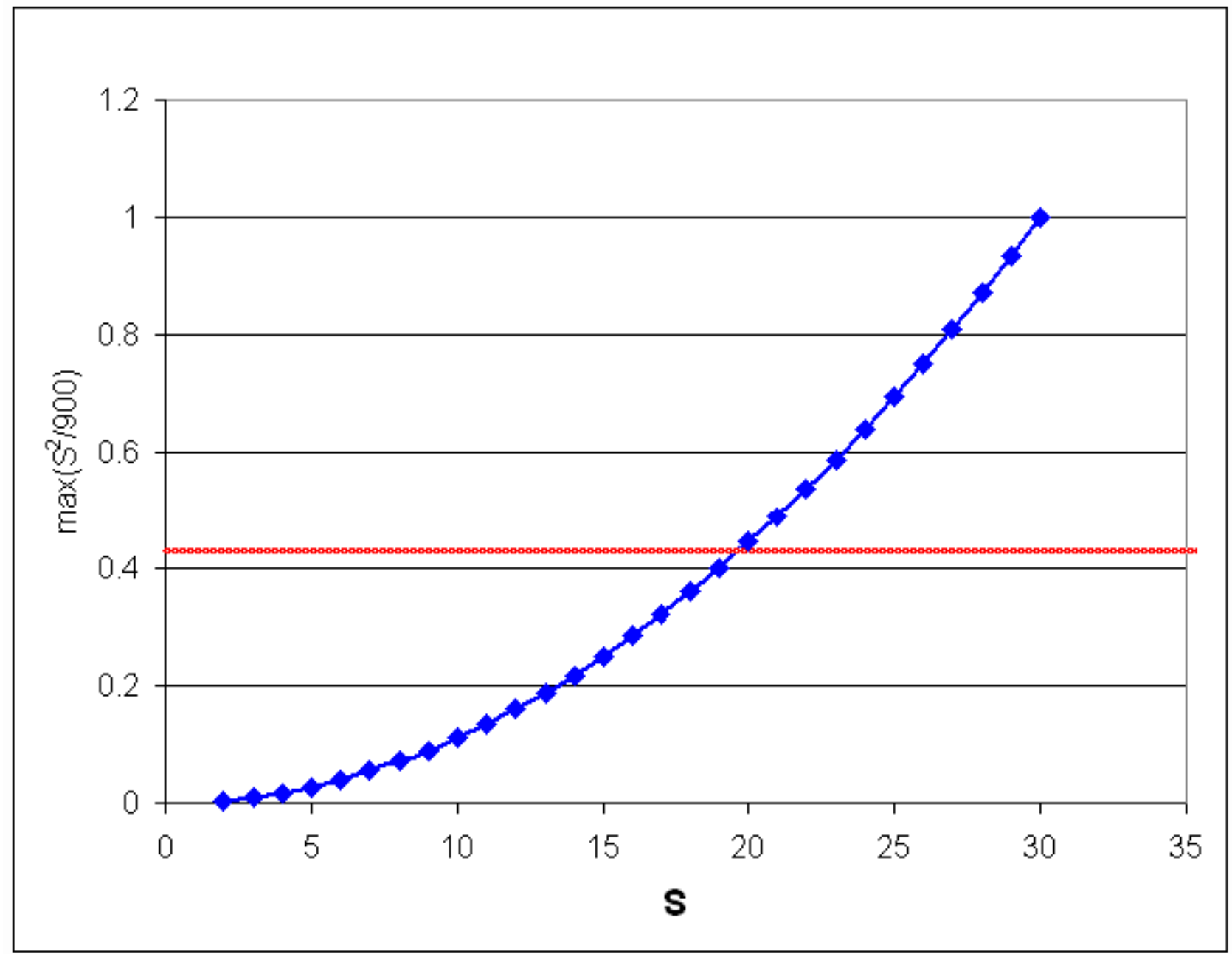

Fig. 4. Peak income value $\left(\mathrm{S}^{2} / 900\right)$ reached by a person with a capability to earn money $\mathrm{S}$. The earning means size is constrained to be the same $\mathrm{L}=\mathrm{S}$. The Pareto distribution threshold is 0.43 . Less than one third of the population can eventually reach the Pareto threshold. 


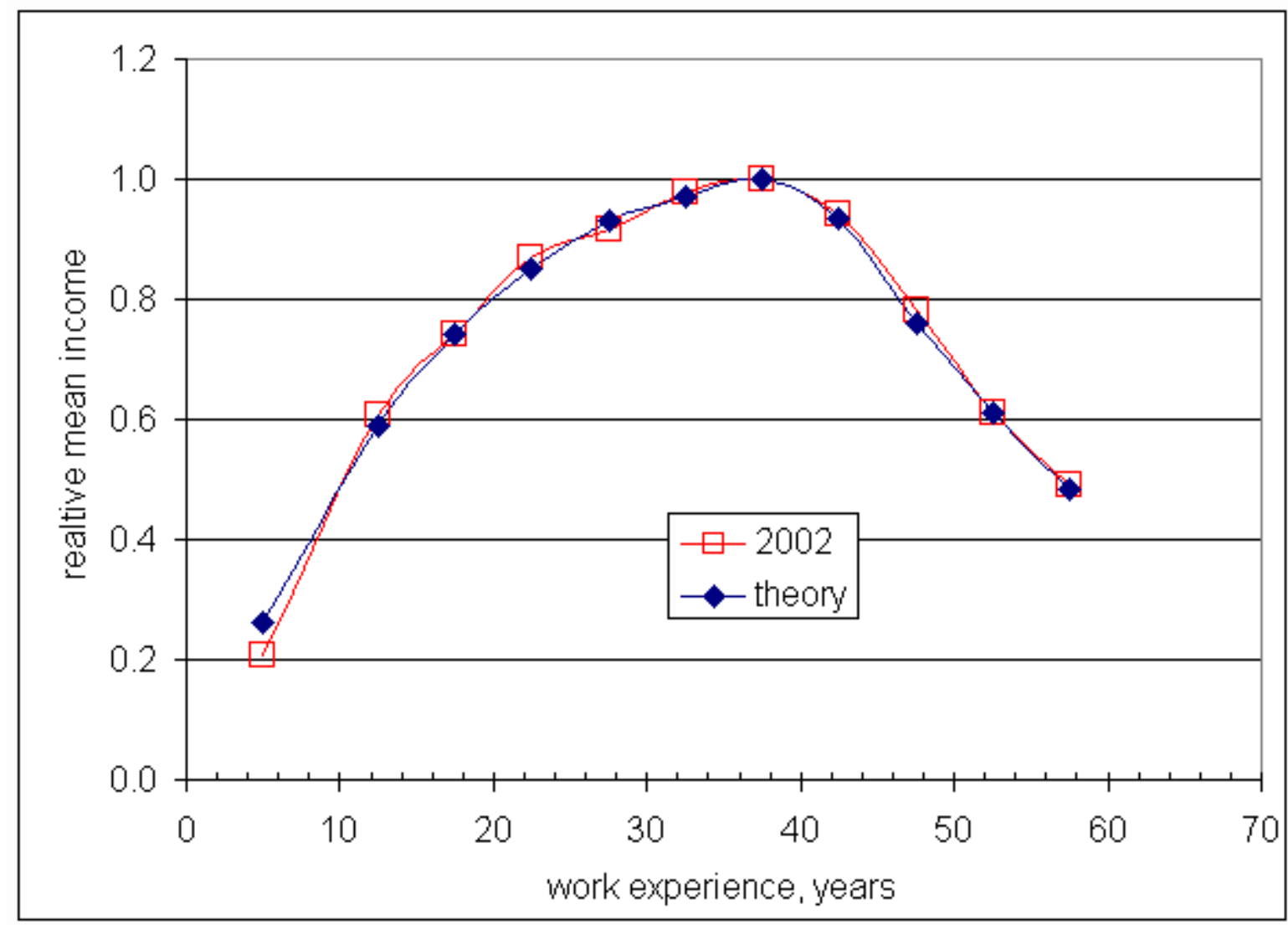

Fig. 5. Interpolation of the mean income distribution for 2002 by two exponential functions. See text for details. 


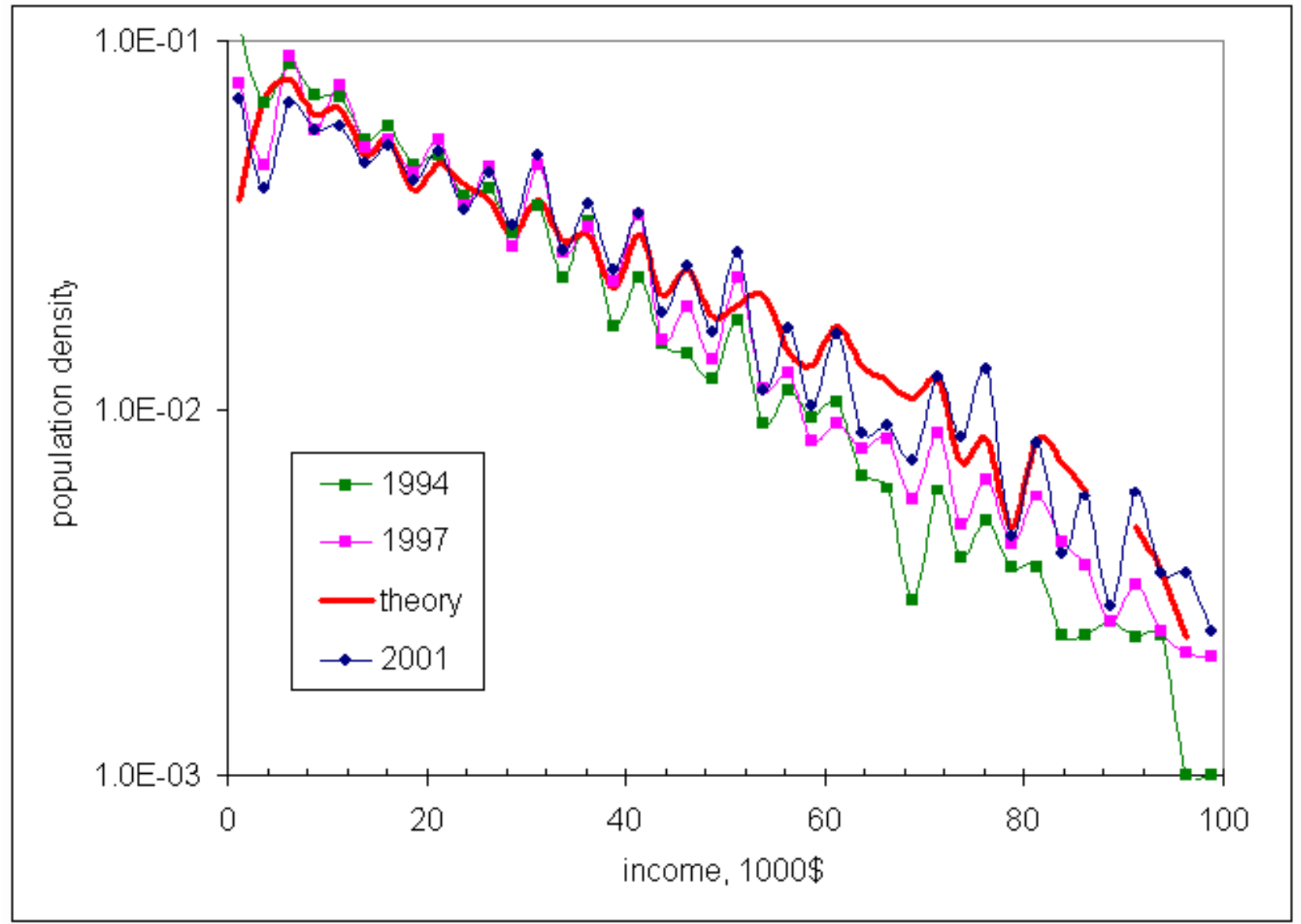

Fig.6. Comparison of a theoretical PID with S and L distributed evenly between 2 and 30 and the observed for the years 1994, 1997, and 2001. 